\title{
Targeted next generation sequencing identified novel mutations in RPGRIP1 associated with both retinitis pigmentosa and Leber's congenital amaurosis in unrelated Chinese patients
}

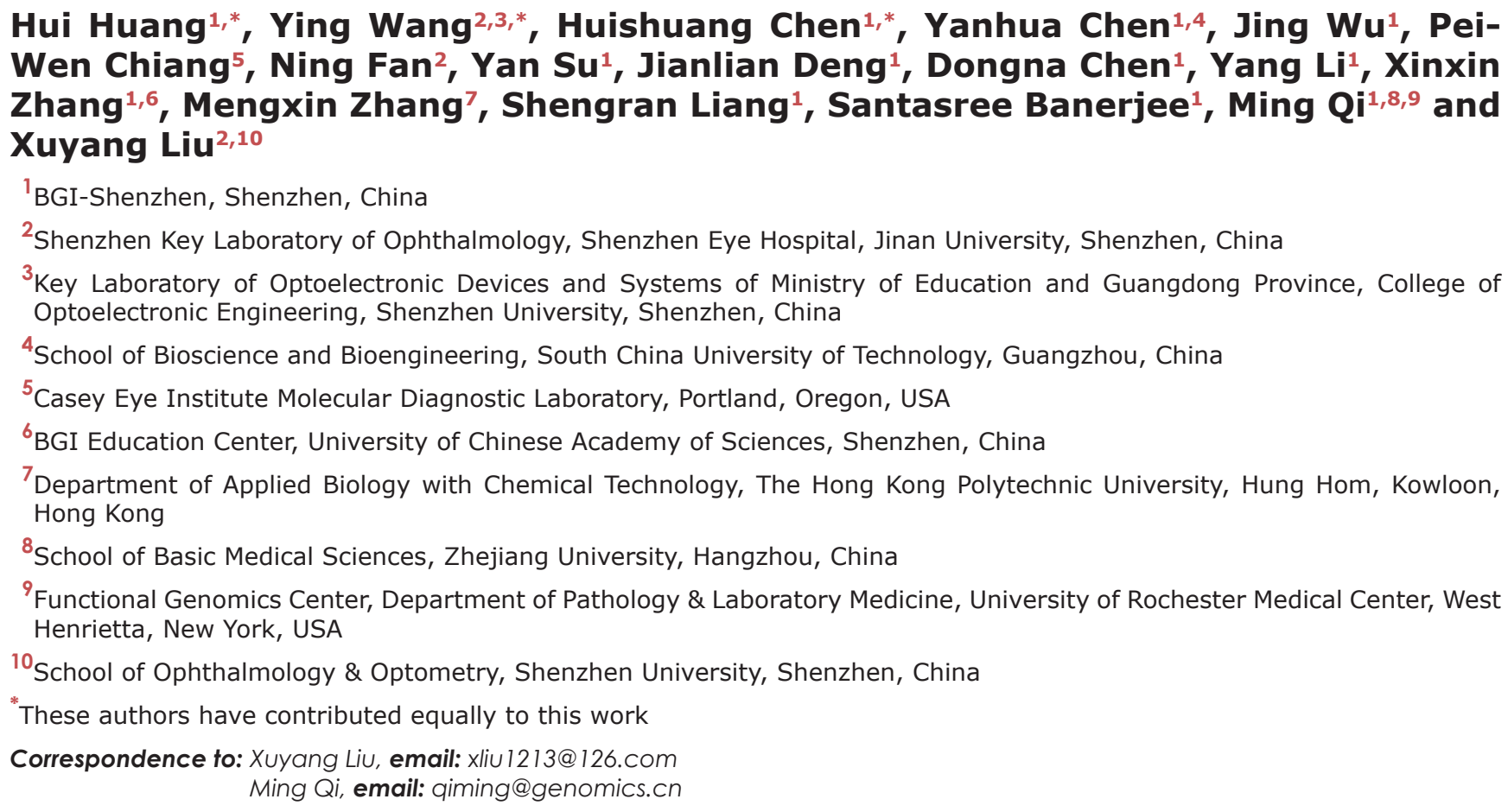
Keywords: targeted next generation sequencing, gene panel, novel mutation, retinitis pigmentosa, Leber's congenital amaurosis Received: October 26, 2016 Accepted: March 15, 2017 Published: April 12, 2017

Copyright: Huang et al. This is an open-access article distributed under the terms of the Creative Commons Attribution License (CC-BY), which permits unrestricted use, distribution, and reproduction in any medium, provided the original author and source are credited.

\section{ABSTRACT}

As the most common inherited retinal degenerations, retinitis pigmentosa (RP) is clinically and genetically heterogeneous. Some of the $R P$ genes are also associated with other retinal diseases, such as LCA (Leber's congenital amaurosis) and CORD (cone-rod dystrophy). Here, in our molecular diagnosis of 99 Chinese RP patients using targeted gene capture sequencing, three probands were found to carry mutations of RPGRIP1, which was known to be associated with pathogenesis of LCA and CORD. By further clinical analysis, two probands were confirmed to be RP patients and one was confirmed to be LCA patient. These novel mutations were co-segregated with the disease phenotype in their families. Our result not only expands the mutational spectrum of the RPGRIP1 gene but also gives supports to clinical diagnosis and molecular treatment of RP patients.

\section{INTRODUCTION}

Retinitis pigmentosa (RP) is the most common inherited retinal dystrophy, affecting approximately 1 in 5,000 individuals worldwide $[1,2]$. RP is primarily associated with the rod photoreceptors, while the cone cells are compromised as the disease progresses [3]. RP individuals exhibit degeneration of photoreceptors or the retinal pigment epithelium (RPE) of the retina in the initial stages of the disease, followed by a progressive reduction 
in the visual field and visual loss [3]. Other ocular findings include atrophic changes in the photoreceptors and RPE followed by the appearing of melanin-containing structures in the retinal vascular layer [3]. The fundus changes include a pale optic nerve, attenuated retinal vessels, and bone spicule-like pigmentation in the mid-peripheral retina [3].

$\mathrm{RP}$ is phenotypically and genetically heterogeneous with almost 60 different genes identified till now, and could be divided into the early onset RP which exhibits symptoms around 2 years old and the late onset RP which may not develop symptoms until mid-life [2]. The clinical distinction between the subtypes of RP, however, is not always clear and there are largely overlapped in phenotype between RP, LCA (Leber's congenital amaurosis), COD or CORD (cone or cone-rod dystrophy) and other retinal diseases [3]. Also, there are other non-RP diseases caused by mutations in the same genes involved with RP. With the development of sequencing technology, more disease-causing genes have been identified, 60 for RP, 20 for LCA and 29 for CORD respectively. Among them, 9 genes (CRB1, CRX, LRAT, PRPH2, RDH12, RPE65, SPATA7, TULP1, IMPDH1) were previously reported to be associated with both RP and LCA (RetNet). However, large number of RP patients is waiting for uncovering their specific genetic underlying causes. In our recent molecular screening of 99 RP patients, two RP and one LCA patients were found to carry mutated RPGRIP1, which was previously reported to be implicated in LCA and CORD $[4,5]$.

\section{RESULTS}

\section{Clinical findings}

Patient P065 is a 30 years old male with early-onset RP. He exhibited early-onset bilateral blurred vision in childhood (visual acuity: 20/25), nystagmus and night blindness at about 23 years old based on his recall. Then he gradually presented with decreasing vision and loss of peripheral visual fields. At 30 years of age, his best corrected visual acuity (BCVA) was 20/133 with -2.25 diopter in the right eye and 20/200 with -2.00 diopter in the left. Fundus examination revealed waxy disc, obviously attenuated retina vascular. No significant pigmentary changes of salt and pepper or bone corpuscle type were noted in the left eye. A few patchy pigmentary could be seen inferior in the periphery retina (Figure 1a). Center $30^{\circ}$ visual field examination (Topcon SBP-300) showed tubular vision with little center visual island left (Figure 1b). Timedominant optical coherence tomography (TD-OCT) showed significant thinner outer retina, especially in the left eye (Figure 1c). Fundus fluorescence angiography (FFA) showed a significant delay in the arm-retina circulation time (ART) from $10 \sim 15 \mathrm{sec}$ to $17 \mathrm{sec}$ of the left eye, which exhibited considerable attenuation of the retinal arterioles. Absent peripheral background fluorescence indicated that choriocapillaris were atrophied. Patchy blocked fluorescence existed in the corresponding pigmentary area in the inferior periphery retina of the right eye. The normal structure of macular arch was absent (Figure 1d). Full-field electroretinography (fERGs) disclosed no detectable rod responses to single flashes of blue light and cone responses to the $30 \mathrm{~Hz}$ flicker that were reduced $99 \%$ and delayed (Figure 1e \& Supplementary Figure 1). All the status of severe retinal structure abnormity and function loss support the clinical diagnosis of RP.

Patient P024, as diagnosed with RP is a 22 years old female with progressive vision weakness for $20+$ years. She is the only patient in a non-consanguineous family. Nystagmus was noticed in her first 8 months, and then gradual loss of visual acuity and night blindness appeared. In the last 6 years, her visual field showed losses of peripheral vision, and eventually became tunnel vision in the age of 21. Her BCVA was 12/400 with both eyes. The dilated fundus examination showed attenuated retina vascular with absent pigmentary changes (Figure 1f). The ERG performed extinguished waveform, which indicated that the rod cell and cone cell were severely damaged (Figure 1g).

Patient P030 is female, 36 years of age, who was diagnosed as LCA. She was born with severe visual impairment and nystagmus without any other symptoms. Her BCVA was hand motion in the age of 36. Dilated fundus examination revealed narrowed arterioles and tortuous veins. Obviously bone corpuscle types pigmentary were seen in the periphery retina (Figure 1h). Scotopic ERG (rod response) after 30min dark adaptation showed the extinguishment of the a-wave and b-wave in both eyes. Only three waves of oscillatory potential ERG were recorded, their amplitudes were severe declined. Cone system was affected with slightly delayed b-wave implicit time. He exhibited the absence of normal ERG response to a $30 \mathrm{~Hz}$ flickering light (Figure 1i). Fundus fluorescence angiography (FFA) showed the edges of optic disks were vague. The illumination of periphery scattered transmitted fluorescences was gradually increasing. Patchy blocked fluorescence existed in the periphery area. Vessels obstruction induced massive periphery nonperfusion area (Figure 1j).

\section{Molecular diagnosis and validation}

We performed next generation sequencing on these 3 patients using the panel described in Methods section. 466,362 , and 351 fold of depth, 94.26\%, 94.31\%, and $94.04 \%$ of coverage were obtained from the 3 patient samples (Supplementary Table 1), respectively, indicating that sufficient depth and coverage were obtained for variants calling. We identified $8,15,8$ rare variants (rare: frequency $<0.01$ in 1000genome, dbSNP, HGMD and our 200 control local database) that would lead to protein coding change (including potential splice site in 10bp of exon/intron borders) in the 283 eye disease related genes (Supplementary Table 2). 
We found these 3 patients carrying mutations not in RP related genes but in gene RPGRIP1 which was previously reported to cause Leber's congenital amaurosis [4] and Cone-rod dystrophy [5]. Among them, one patient carried compound heterozygous mutations and 2 patients carried homozygous mutations (Table 1). Sanger sequencing and quantitative PCR was undertaken to validate all these 4 RPGRIP1 mutations in the proband and their family members (Figure 2).

Patient P065 was detected to carry one novel homozygous splicing site mutation c.1468-2A $>\mathrm{G}$ in gene RPGRIP1, his parents were consanguineous with normal phenotype. This homozygous mutation was not recorded in the HapMap and $1 \mathrm{~K}$ genome and was co-segregated with the disease phenotype in the family; the normal family members carried only one heterozygous mutant allele (Figure 2a \& Figure 2b).

This RPGRIP1 mutation disrupts the splice acceptor site of exon 12 . With this type of splicing defect, it would be desirable to determine the consequences at the mRNA level by reverse transcription-PCR (RT-PCR) of RPGRIP1 cDNA. Partial genomic DNA constructs consisting of exons 12 and partial intron 11 and intron 12 of RPGRIP1, with or without the splice site mutation, were expressed in COS-7 cells. RT-PCR and direct sequencing of RPGRIP1 cDNA from cells transfected with wild-type construct (a)

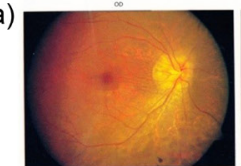

(b)

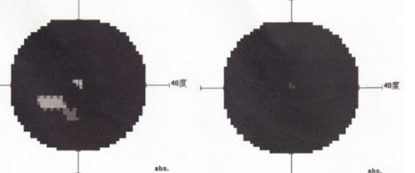

(c)

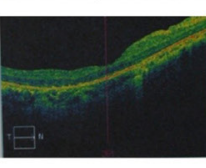

(d)
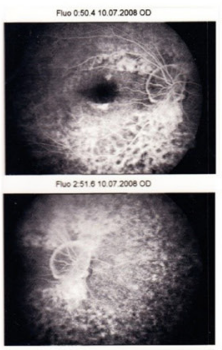

(e)

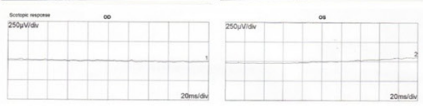

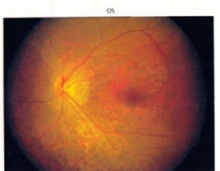

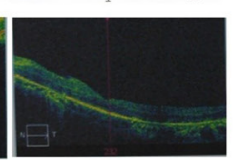

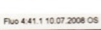
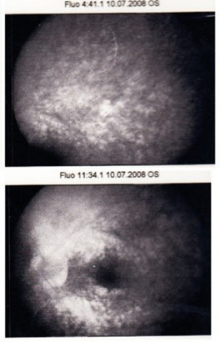

mone
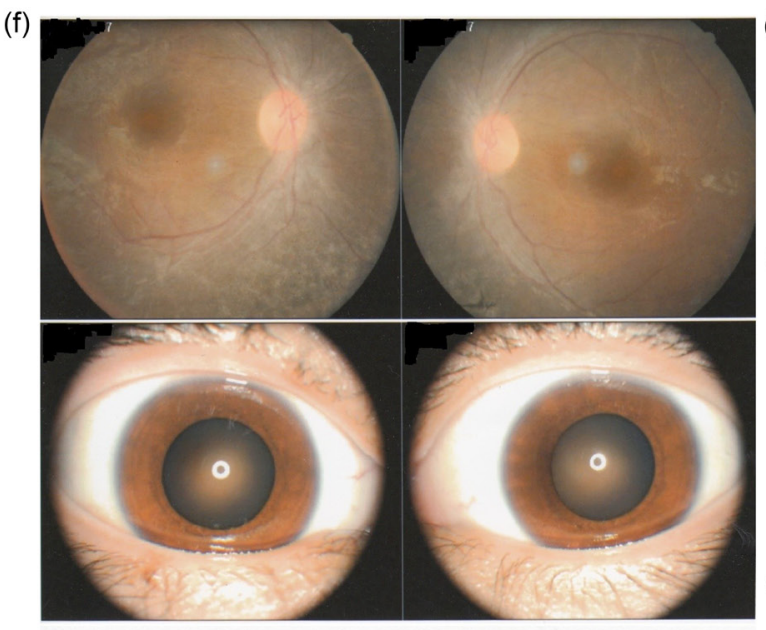

(g)

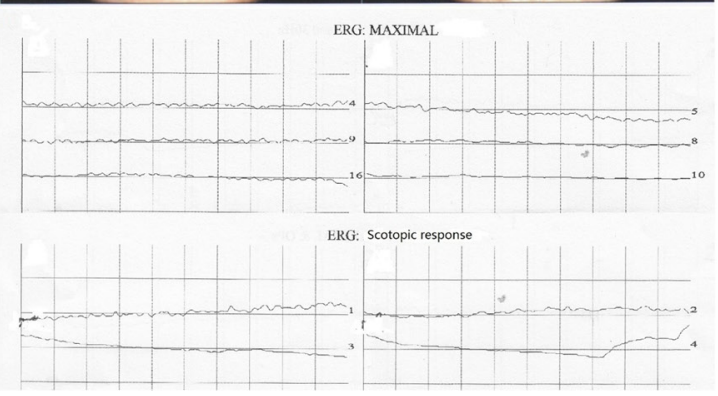

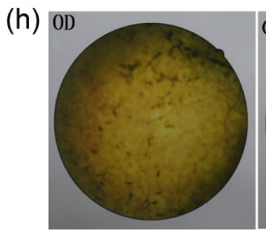

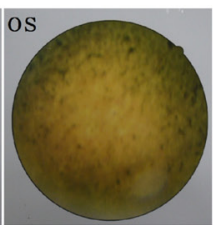

(i)
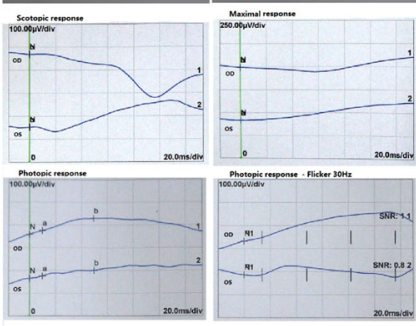

(j)
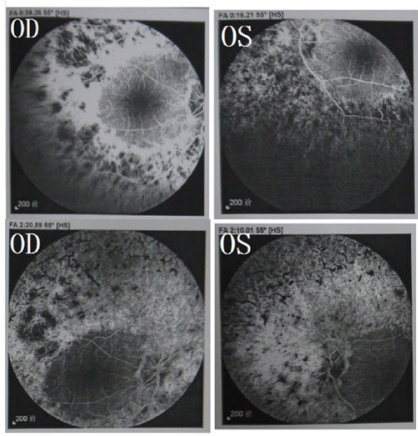

Figure 1: Clinical characteristics of 3 patients Patient P065. (a) Fundus photographs showed disc pallor with wax yellow. Obviously attenuated narrow arterioles and tortuous veins lay on the orange background of the retina. (b) vision field examination (Topcon SBP-300) show tubular vision with litter center visual island. (c) OCT showed significant thinner of outer layer of retina, especially in the left eye. (d) Fudus fluorescence angiography (FFA) showed a significant delay in the arm-retina circulation time (ART) from 10 15sec to $17 \mathrm{sec}$ of the left eye. There were uniform scattered dotted transmitted fluorescences on the both side in the late phase. Absent peripheral background fluorescence indicted choriocapillaris were atrophy. Patchy blocked fluorescence existed in the corresponding pigmentary area in the inferior periphery retina of the right eye. The normal structure of macular arch was absent. (e) Scotopic ERG (rod response) and dark adapted bright flash ERG showed a-, b-waves extinguished in both eyes. The patient exhibited extinguished in the ERG response. Patient P024 (f) Fundoscopy revealed narrowing of retinal vessels. Scattered patchy dislocatedpigmentary changes could be seen in the periphery retina of both eyes. (g) ERG revealed extinguished waveforms. Patient P030 (h) Dilated fudus examination revealed arterioles and tortuous veins narrowed. Patient's optic disk was sanguineness. Obviously bone corpuscle types pigmentary were seen in the periphery retina. (i) Scotopic ERG (rod response) after 30min dark adaptation showed the extinguishment of waveform in both eyes. B-wave of photopic ERG (cone response) after 10min light adaptation showed implicit time delay to 49ms which was amplified to severe decline in both eyes. Patient exhibited the absence of nomal ERG response to a $30 \mathrm{~Hz}$ flickering light. (j) FFA showed Retinal arterioles were narrowed. The edges of optic disks were vague. The illumination of periphery scattered transmitted fluorescences was increasing gradually. Patchy blocked fluorescence existed in the periphery area. Vessels obstruction induced massive periphery non-perfusion area. 
Table 1: RPGRIP1 mutations identified in three families

\begin{tabular}{|c|c|c|c|c|c|}
\hline Patient ID & Disease & Type & Exon & Mutation & Protein change \\
\hline 65 & ARRP & Homozygous & Intron11 & c. $1468-2 A>G$ & Exon 12 del \\
\hline 24 & ARRP & $\begin{array}{c}\text { Compound } \\
\text { heterozygous }\end{array}$ & Exon 2 / Exon 14 & $\begin{array}{l}\text { c. } 154 \mathrm{C}>\mathrm{T} / \\
\text { c. } 2020 \mathrm{C}>\mathrm{T}\end{array}$ & $\begin{array}{c}\text { p.Arg52*/ } \\
\text { p.Pro674Ser }\end{array}$ \\
\hline 30 & LCA & Homozygous & Exon1-22 & ex1-22del & \\
\hline
\end{tabular}

RP: Retinitis Pigmentosa; LCA: Leber congenital amaurosis.

showed normal splicing of exon 12. In contrast, direct sequencing of mutant RT-PCR products revealed complete loss of exon 12 resulting removal of 48 amino acids in the RPGRIP1 polypeptide due to abolition of the acceptor splice site (data not shown). Prediction of the abolition effect of this splice site was made using a splice predictor program, Human Splicing Finder (http://www.umd.be/ HSF). This prediction gave a potential breakage of splice site (ttcctctacggCC), compared with a probability of 92.13 for the wild type (ttccetctacagCC) splice site. All the results suggest that aberrant splicing caused by this mutation is quite efficient as it completely abolishes normal splicing in the mutant allele.

Patient P024 carried one nonsense mutation c. $154 \mathrm{C}>\mathrm{T}$ (p. Arg52*) and one novel missense mutation c. $2020 \mathrm{C}>\mathrm{T}$ (p. Pro674Ser) in gene RPGRIP1. Mutation p. Arg52* was inherited from the father and mutation c.2020C $>$ T (p. Pro674Ser) came from the mother (Figure 2c \& Figure 2d). The nonsense mutation p. Arg52* was previously reported in a retinitis punctata albescens (RPA) patient [6]. It creates premature stop codons and is likely to be a null allele. The mutation $\mathrm{p}$. Pro674Ser is novel and not recorded in the HapMap and $1 \mathrm{~K}$ genome database or our local database, it changed a proline (non-polar, uncharged amino acid) into a serine (polar) in the protein. In addition, it was predicted to be "probably damaging" with a score of 1.000 using PolyPhen-2 (http://genetics.bwh.harvard.edu/pph2/). The proline at position 674 is present as a highly conserved amino acid among mammals (Figure 2e). It is highly likely that these mutations are the primary cause of the retinal disease in this patient.

Patient P030 was found carrying no SNV/InDel mutation, so we tried to search a CNV (copy number variantion) from exonic depth and coverage in retinal genes, and a large homozygous deletion of exon 1-22 in RPGRIP1 was found (Supplementary Figure 2), which was inherited from her healthy parents separately (Figure 2f). The subsequent quantity PCR confirmed this mutation (Figure $2 \mathrm{~g}$ ). This large deletion may lead to absence of RPGRIP1 protein which interacts with RPGR in the connecting of cilia of rods and cones [7]. This homozygous mutation was not identified in the HapMap and 1000 genome and was cosegregated with the disease phenotype. Her normal brother didn't carry the mutation (Figure $2 \mathrm{~g}$ ).

\section{DISCUSSION}

In this study, we found $2 \mathrm{RP}$ patients and $1 \mathrm{LCA}$ patient carrying mutations in RPGRIP1 using our panel NGS methods. One RP patient carried a novel homozygous mutation of c. $1468-2 \mathrm{~A}>\mathrm{G}$ in intron 11 of RPGRIP1, resulting in the formation of alternative transcripts and leading to the complete loss of exon 12 (data not shown). Defects in either RPGRIP1 or its RPGR interacting protein probably alter a functional complex in the connecting cilia of rods and cones. Another RP patient carried compound heterozygote for c.154C $>$ T (p. Arg52*) and c.2020C $>$ T (p. Pro674Ser). The LCA patient carried a large homozygous deletion that extends from exon 1 to intron 22 in RPGRIP1. All these mutations were confirmed to be co-segregated with disease phenotype in respective families by Sanger sequencing or quantitative PCR.

This study provides evidence supporting that RPGRIP1 is a new RP-causing gene by finding mutations in 2 unrelated cases. We also found that the LCA patient carrying mutation in RPGRIP1 presented with similar phenotype. In clinical signs and symptoms, all these 3 patients presented with early-onset night blindness and low visual acuity. According to the clinical examination and genetic test of these 3 patients, it suggested a correlation between disease severity and the nature of the mutations. Patient P030 carrying a large exon1-22 deletion mutation in RPGRIP1 showed the most severe phenotype of three patients, she was blind with light perception at birth and her current BCVA was hand motion, and she was diagnosed with LCA. Patient P024 and P065, who were diagnosed with RP, showed slighter symptoms than Patient P030. Patient P024 carrying a nonsense ( $p$. Arg52*) and a missense mutation ( $p$. Pro674Ser) showed a worse BCVA comparing to Patient P065 who carried a homozygous splice site mutation (c.1468-2A $>$ G). It is possible that the large proportion of coding region deletion and nonsense mutation in the initiating terminal coding region leads to loss of the whole protein, while the missense mutation and the splice site mutation (although it resulted in the complete loss of exon 12) retains most of protein residue.

The RPGRIP1 gene contains 24 exons and encompasses three main domains that are RPGR interacting domain (RID), coiled-coil (C2) domains and 
a domain of unknown function. The RPGRIP1 protein is localized in the connecting cilia of human cone and rod photoreceptors that connect inner and outer segments, where it binds RPGR to the cilium. Mice lacking RPGRIP elaborate grossly oversized outer segment disks resembling a cytochalasin D-induced defect and have a more severe disease than mice lacking RPGR. Mice lacking both proteins are phenotypically indistinguishable from mice lacking RPGRIP alone [8]. RPGRIP1 has an interaction not only with RPGR, but also with NPHP4 and SDCCAG8, Rpgrip $1^{\mathrm{nm} 247}$ mice without RPGRIP1 expression lack NPHP4 and RPGR in photoreceptor cilia, whereas the SDCCAG8 and acetylated-a-tubulin ciliary localizations are strongly decreased, even though the NPHP4 and SDCCAG8 expression levels are normal and those of acetylated-a-tubulin and c-tubulin are upregulated [9]. Mutations in NPHP4 and SDCCAG8 would cause Senior-Loken syndrome and Bardet-Biedl syndrome, these two disorders are characterized by LCA/RP and other systemic features including kidney conditions [10-11]. These studies suggest that RPGRIP1 is important in visual pathway and has an essential function in eye development, and when mutated, it may cause RP disease.

Interestingly, RP is both clinically and genetically heterogeneous. The phenotype of such patients is overlap between retinal diseases, as RP and LCA is more overlap in the initial stage, while RP and CORD is in the end stage [3]. There are nine genes (CRB1, CRX, LRAT, PRPH2, RDH12, RPE65, SPATA7, TULP1, IMPDH1) overlap between RP and LCA, eight genes (CRX, PROM1, PRPH2, SEMA4A, ABCA4, C8orf37, CERKL, RPGR) between RP and COD/CORD (RetNet: https://sph.uth. edu/retnet/sum-dis.htm). $C R X$ and PRPH2 are the only two genes associated with RP, LCA and CORD. Carlo Rivolta et al. analyzed 18 mutations and Huang L et al. also reviewed 49 mutations in $C R X$, but they all could not
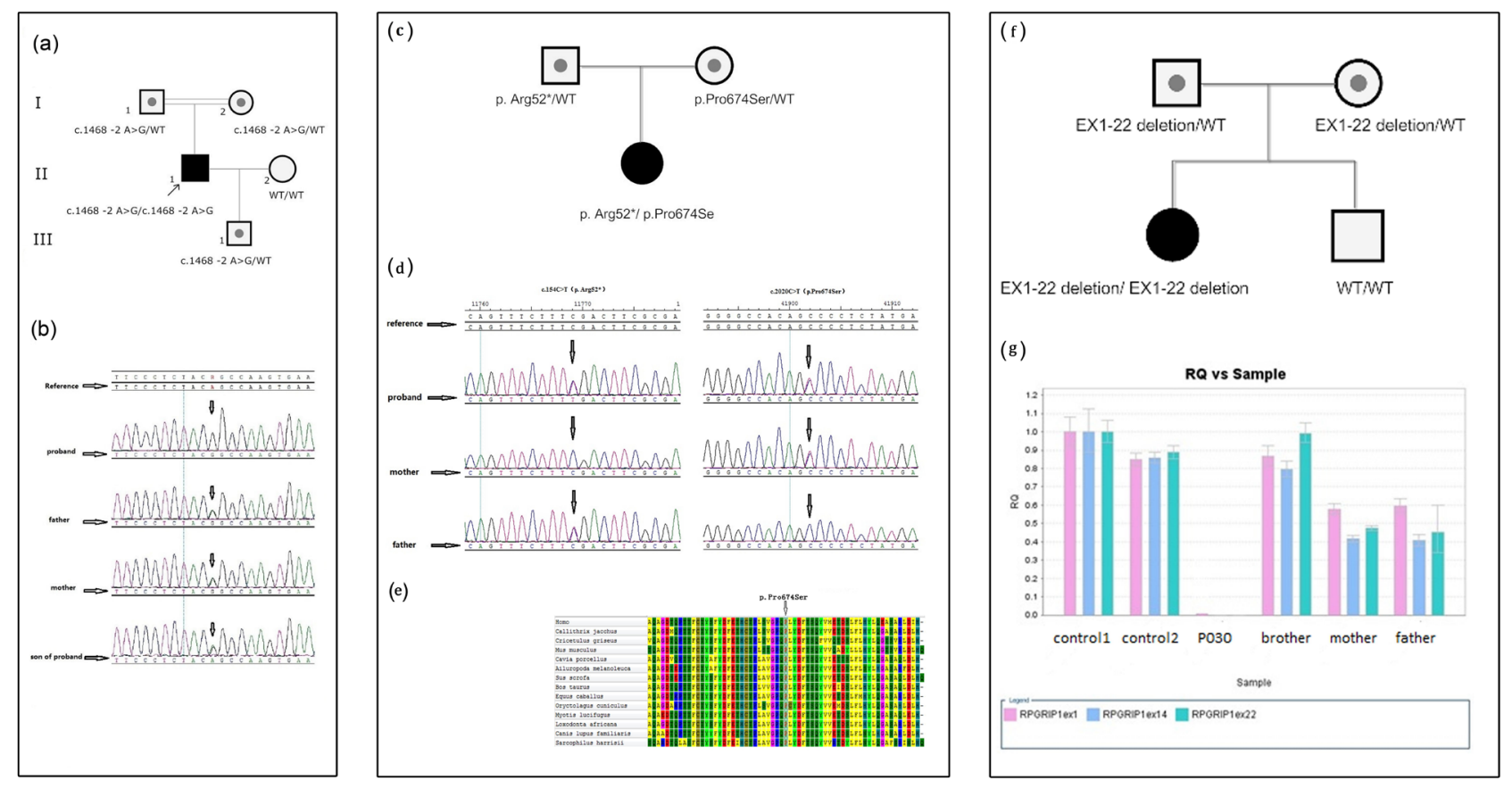

Figure 2: Molecular genetic test. (a) The pedigree of Patient P065; (b) Sanger sequence chromatograms for the pedigree of Patient P065; (c) The pedigree of patient P024; (d) Sanger sequence chromatograms for the pedigree of Patient P024; (e) Evaluation of the novel RPGRIP1 missense mutation in Patient P024. Multiple alignments using MEGA6 software (Tamura K et al. 2013) and amino acid conservation of the novel missense sequence variants were performed. The alignment results showed that proline at codon 2020 were fully conserved through all species. (f) The pedigree of patient P030; (g) Quantity PCR for the large deletion in pedigree of Patient P030.

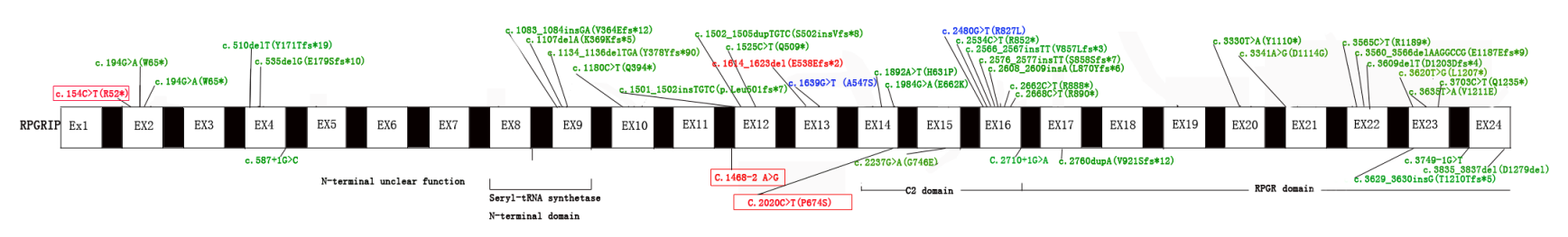

Figure 3: Reported variants in RPGRIP1 identified in individuals with LCA, CORD and RP. 
uncover any correlation between phenotype and genotype [12-13]. In this study, we also summarized the mutations in RPGRIP1 which were reported to be related to LCA or CORD as many as we can (Figure 3 ). We can see that approximately $90 \%$ of the reported mutations causing LCA in RPGRIP 1 were frameshift or nonsense mutations that will lead to create a prematurely truncate protein, which are expected to abolish the function of RPGR interacting domain, even the RPGRIP1 function. RPGRIP1 was found to cause LCA in 2001 [4], then in 2002, Cremers FP et al. deduced that mutations allow residual RPGRIP1 activity may result in less severe phenotypes, such as RP or CORD, than LCA [14]. Subsequently in 2003, it was proved that homozygous missense mutations in RPGRIP1 would cause CORD [5]. In 2005, J C Booij et al. found two RP patients carrying respective frameshift and missense mutations in RPGRIP1, but it's a pity that they identified only one heterozygous mutation in every patient [15]. Yet, in this study, we found two RP patients caused by homozygous splice site mutation and compound heterozygous (missense and nonsense) mutations in RPGRIP1. Taken together, it showed strong evidence for the conclusion that RPGRIP1 is a causative gene for RP.

In the past, people always found new diseasecausing genes by linkage study and subsequent whole exome sequencing (WES). In this study, we found a new RP disease-causing gene by panel sequencing. Although all the captured genes were known hereditary eye diseasecausing genes, many different retinal disease genes are in the same pathway (KEGG), it provides the potential possibility that one gene in this pathway causes several retinal diseases. So we can find a new gene for some retinal diseases using a panel that capture genes in the pathway.

In conclusion, we reported that the mutations in RPGRIP1 causes early-onset RP based on the data from panel NGS, co-segregation, OCT, histopathological and electrophysiological analysis. Thus our findings suggest that RPGRIPl gene can also be classified into the RP causative genes, that expands the clinical spectrum of RPGRIP1 related disease. Since the two RP patients in this study were found in a cohort of 99 unselective Chinese patients, we can expect that RPGRIP1 would cause about $2 \% \mathrm{RP}$ patients in Chinese population. In addition, RPGRIP1 has been reported with effective treatment in animal models and showing molecular treatment potential in a LCA patient $[16,17]$. Hope our research could improve the diagnosis and treatment of RP and LCA.

\section{MATERIALS AND METHODS}

\section{Patients}

This study was performed in agreement with the declaration of Helsinki. All the patients and their legal representatives have given permission for the mutation detection. Informed consents were also obtained from patients. This study was approved by the institutional review board on bioethics and biosafety of BGI, and the IRB approval number is BGI-IRB 14002.

\section{DNA preparation}

Venous blood samples were obtained from the probands and their family members. Genomic DNA was extracted from $200 \mu$ peripheral venous blood by standard procedures using QIAmp DNA Blood Mini kit (QIAGEN, Hilden, Germany) according to recommended instructions. DNA integrity was evaluated by $2 \%$ agarose gel electrophoresis. All DNA samples were stored at $-20^{\circ} \mathrm{C}$ after the analysis with NanoDrop 2000 (Thermo Fisher Scientific, Waltham, USA).

\section{Panel sequencing library preparation and sequencing}

283 genes, which include 164 known retinal diseasecausing genes, were collected by systematic database (GeneReviews( $\left({ }^{\circ}\right)$, OMIM, and RetNet), literature searches, and expert reviews (Supplementary File 1). Customized oligonucleotide probes were designed to capture exons and adjacent $30 \mathrm{bp}$ sequences of above genes using NimbleGen (Roche) online oligonucleotide probe design system. Target sequencing libraries were prepared as below: $1 \mu \mathrm{g}$ genomic DNA was sonicated to $200 \sim 300$ bp fragments, followed with end-repair, A-tailing, Illumina adaptors ligation, 4 cycles of pre-capture PCR amplification and sample indexing. Then the indexed PCR product of 20-30 samples were pooled, targeted capture was performed by hybridizing with capture probes, and followed by 15 cycles of PCR amplification. DNA sequencing was done on Illumina HiSeq2500 sequencer to generate paired-end reads including $90 \mathrm{bps}$ at each end and $8 \mathrm{bps}$ of the index tag. And bases were called by the Illumina build-in Pipeline.

\section{Bioinformatics analysis and data filtering}

Reads filtering was surveillance by indexed primers. The reference genome was downloaded from the NCBI, version GRCh37 (hg19). Sequence was aligned to the reference using Burrows Wheeler Aligner (BWA) MultiVision software package [18]. SNVs were called using SOAPsnp [19], while small InDels $(<20 \mathrm{bp})$ were called using the Samtools (Tools for alignments in the SAM format) Version: 0.1.18, http://samtools.sourceforge.net/. The threshold for filtering SNPs included the followings: 1) the consensus quality score had to be $>=20$ (The quality score is a Phred score, generated by the program SOAPsnp, quality score 20 represents $99 \%$ accuracy of a base call); 2) the number of uniquely mapped reads supporting a SNP had to be $>=4 ; 3$ ) the estimated copy 
number is no more than 2 ; 4) the distance between two SNPs should be larger than 5 .

\section{Sanger sequencing}

Mutations identified in Target sequencing were validated by Sanger sequencing. Primers flanking the candidate loci were designed based on reference genomic sequences of Human Genome from gene bank in NCBI (GRCh37, hg19) and synthesized by Invitrogen (Shanghai, China). PCR amplification was carried out in ABI 9700 Thermal Cycler. Subsequently, all PCR products were sequenced on ABI PRISM 3730 automated sequencer (Applied Biosystems).

\section{Minigene construction and exon-trapping}

To determine the function of the mutation at transcriptional level, in vitro exon trapping studies were performed. Using DNA from proband as a template, harboring a homozygous mutant allele, a genomic fragment, containing $315 \mathrm{bp}$ of intron 11, exon 12 and $312 \mathrm{bp}$ of intron 12 with both 5 ' and 3 ' intronic flanking sequences, was generated and cloned into pSPL3 (Invitrogen, Carlsbad, CA) exon trapping vector through double digestion by BamHI and XhoI. WT and mutant plasmids were transiently transfected into COS7 (African green monkey kidney fibroblast-like) cell line using lipo2000 (Invitrogen, Carlsbad, CA). COS-7 cells were cultured in Dulbecco's modified Eagle's medium supplemented with $10 \%$ fetal bovine serum, $1 \%$ penicillinstreptomycin, and $1 \%$ glutamine in a humidified, $5 \% \mathrm{CO} 2$ incubator at $37^{\circ} \mathrm{C}$. At $48 \mathrm{~h}$ post transfection, total RNA was extracted with Trizol (TaKaRa, Dalian, China). cDNA was prepared using $5 \mu \mathrm{g}$ total RNA in a total volume of $20 \mu \mathrm{l}$ with superscript II RNAse H-reverse transcriptase and oligo-dT priming (TaKaRa, Dalian, China). Amplification products obtained by PCR with vector primers SD6 (5'-TCTGAGTCACCTGGACAACC-3') and SA2 (5'-ATCTCAGTGGTATTTGTGAGC-3'), with recommended PCR reaction condition, were separated on a $2 \%$ TBE agarose gel. Amplification products were characterized by direct sequencing.

\section{Author contributions}

Ming qi, Xuyang Liu, Santasree Banerjee, Hui Huang design the study. Pei-Wen Chiang performed the molecular diagnosis by Sanger sequencing. Yanhua Chen carried on the bioinformatic analysis. Jing $\mathrm{Wu}$, Yan Su, Jianlian Deng, Huishuang Chen, contributed to the molecular diagnosis analysis based on NGS. Xuyang Liu, Ning Fan and Ying Wang worked on the clinical and genetic studies. Yanhua Chen contributed to the Sanger sequencing validation and quantitative PCR experiments. Huishuang Chen, Hui Huang, Ying Wang wrote the paper. All authors read and approved the final manuscript.

\section{ACKNOWLEDGMENTS}

This work was supported by grants from the National Natural Science Foundation of China (NSFC81500718 and 81371009), the Science and Technology Innovation Committee of Shenzhen (JCYJ20160428144701106) and Shenzhen Municipal Science and Technology Plan Project (No.201402089, 201507044). We thank all the patients and their families for their participation.

\section{CONFLICTS OF INTEREST}

The authors declare no conflicts of interest.

\section{REFERENCES}

1. Haim, M. Epidemiology of retinitis pigmentosa in Denmark. Acta Ophthalmol Scand Suppl. 2002; 233: 1-34.

2. Chang S, Vaccarella L, Olatunji S, Cebulla C, Christoforidis J. Diagnostic challenges in retinitis pigmentosa: genotypic multiplicity and phenotypic variability. Curr Genomics. 2011; 12: 267-75.

3. Neveling K, den Hollander AI, Cremers FP, Collin RW. Identification and analysis of inherited retinal disease genes. Methods Mol Biol. 2013; 935: 3-23.

4. Dryja TP, Adams SM, Grimsby JL, McGee TL, Hong DH, Li T, Andréasson S, Berson EL. Null RPGRIP1 alleles in patients with Leber congenital amaurosis. Am J Hum Genet. 2001; 68: 1295-8.

5. Hameed A, Abid A, Aziz A, Ismail M, Mehdi SQ, Khaliq $\mathrm{S}$. Evidence of RPGRIP1 gene mutations associated with recessive cone-rod dystrophy. J Med Genet. 2003; 40: 616-9.

6. Abu-Safieh L, Alrashed M, Anazi S, Alkuraya H, Khan AO, Al-Owain M, Al-Zahrani J, Al-Abdi L, Hashem M, Al-Tarimi S, Sebai MA, Shamia A, Ray-Zack MD, et al. Autozygome-guided exome sequencing in retinal dystrophy patients reveals pathogenetic mutations and novel candidate disease genes. Genome Res. 2013; 23: 236-47.

7. Hong DH, Yue G, Adamian M, Li T. Retinitis pigmentosa GTPase regulator (RPGRr)-interacting protein is stably associated with the photoreceptor ciliary axoneme and anchors RPGR to the connecting cilium. J Biol Chem. 2001; 276: 12091-9.

8. Zhao Y, Hong DH, Pawlyk B, Yue G, Adamian M, Grynberg M, Godzik A, Li T. The retinitis pigmentosa GTPase regulator (RPGR)- interacting protein: subserving RPGR function and participating in disk morphogenesis. Proc Natl Acad Sci U S A. 2003; 100: 3965-70.

9. Patil H, Tserentsoodol N, Saha A, Hao Y, Webb M, Ferreira PA. Selective loss of RPGRIP1-dependent ciliary targeting of NPHP4, RPGR and SDCCAG8 underlies the degeneration of photoreceptor neurons. Cell Death Dis. 2012; 3: e355. 
10. Otto E, Hoefele J, Ruf R, Mueller AM, Hiller KS, Wolf MT, Schuermann MJ, Becker A, Birkenhäger R, Sudbrak R, Hennies HC, Nürnberg P, Hildebrandt F. A gene mutated in nephronophthisis and retinitis pigmentosa encodes a novel protein, nephroretinin, conserved in evolution. Am J Hum Genet. 2002; 71: 1161-7.

11. Otto EA, Hurd TW, Airik R, Chaki M, Zhou W, Stoetzel C, Patil SB, Levy S, Ghosh AK, Murga-Zamalloa CA, van Reeuwijk J, Letteboer SJ, Sang L, et al. Candidate exome capture identifies mutation of SDCCAG8 as the cause of a retinal-renal ciliopathy. Nat Genet. 2010; 42: 840-50.

12. Rivolta C, Berson EL, Dryja TP. Dominant Leber congenital amaurosis, cone-rod degeneration, and retinitis pigmentosa caused by mutant versions of the transcription factor CRX. Hum Mutat. 2001; 18: 488-98.

13. Huang L, Xiao X, Li S, Jia X, Wang P, Guo X, Zhang Q. CRX variants in cone-rod dystrophy and mutation overview. Biochem Biophys Res Commun. 2012; 426: 498-503.

14. Cremers FP, van den Hurk JA, den Hollander AI. Molecular genetics of Leber congenital amaurosis. Hum Mol Genet. 2002; 11: 1169-76.

15. Booij JC, Florijn RJ, ten Brink JB, Loves W, Meire F, van Schooneveld MJ, de Jong PT, Bergen AA. Identification of mutations in the AIPL1, CRB1, GUCY2D, RPE65, and RPGRIPI genes in patients with juvenile retinitis pigmentosa. J Med Genet. 2005; 42: e67.

16. Pawlyk BS, Smith AJ, Buch PK, Adamian M, Hong DH, Sandberg MA, Ali RR, Li T. Gene replacement therapy rescues photoreceptor degeneration in a murine model of Leber congenital amaurosis lacking RPGRIP. Invest Ophthalmol Vis Sci. 2005; 46: 3039-45.

17. Roman AJ, Boye SL, Aleman TS, Pang JJ, McDowell JH, Boye SE, Cideciyan AV, Jacobson SG, Hauswirth WW. Electroretinographic analyses of Rpe65-mutant rd12 mice: developing an in vivo bioassay for human gene therapy trials of Leber congenital amaurosis. Mol Vis. 2007; 13: 1701-10.

18. Li H, Durbin R. Fast and accurate short read alignment with Burrows-Wheeler transform. Bioinformatics. 2009; 25: 1754-60.

19. Li R, Li Y, Fang X, Yang H, Wang J, Kristiansen K, Wang J. SNP detection for massively parallel whole-genome resequencing. Genome Res. 2009; 19:1124-32. 